\title{
Composición del ensamblaje de aves en una zona periurbana de Medellín, Colombia
}

\author{
Composition of bird assemblages in a periurban area from Medellín, \\ Colombia
}

Agudelo-Rendón D, Rendón-Gutiérrez N, Cadavid-Ramírez AC, Choperena-Palencia MC, AriasMonsalve CS, Gómez-R DA. Composición del ensamblaje de aves en una zona periurbana de Medellín, Colombia. Rev Colombiana Cienc Anim. Recia. 2020; 12(2):e782. https://doi.org/10.24188/recia. v12.n2.2020.782

Universidad de Sucre, Colombia

Los autores permiten a RECIA reimprimir el material publicado en él. En caso de que un autor quiera traducir o usar una publicación parcial o completa de nuestro Diario, el autor debe obtener un permiso por escrito del editor de la revista.

Copyright (C) 2020. El (los) autor (es). Este es un artículo de acceso abierto distribuido bajo los términos de Creative Commons Attribution 4.0 (https://creativecommons.org/licenses/by-ncsa/4.0/), El uso, distribución o reproducción está permitido, siempre que se acrediten al autor original y al propietario del copyright y que se cite la publicación original en esta revista, de acuerdo con la práctica académica aceptada. No se permite el uso, distribución o reproducción que no cumpla con estos términos. 


\section{Composición del ensamblaje de aves en una zona periurbana de Medellín, Colombia}

\section{Composition of bird assemblages in a periurban area from Medellín, Colombia}

Daniela Agudelo-Rendón. MV.

Corporación Universitaria Remington, Facultad de Medicina Veterinaria, DOI: https://doi.org/10.24188/recia.v13.n1.2021.782

Grupo GINVER, Medellín, Colombia.

daniagudelo346@hotmail.com

(D) https://orcid.org/0000-0003-0098-8947

Nataly Rendón-Gutiérrez. MV.

Corporación Universitaria Remington, Facultad de Medicina Veterinaria, Grupo GINVER, Medellín, Colombia.

natisgor2009@gmail.com

(D) https://orcid.org/0000-0002-6400-9659

Ana Cristina Cadavid-Ramírez. M.Sc.

Corporación Universitaria Remington, Facultad de Medicina Veterinaria, Grupo GINVER, Medellín, Colombia.

acadavid@lasallistadocentes.edu.co

(D) http://orcid.org/0000-0003-4423-4223

Mary Cerliz Choperena-Palencia. M.Sc.

Corporación Universitaria Remington, Facultad de Medicina Veterinaria, Grupo GINVER, Medellín, Colombia

mchoperena@uniremington.edu.co

(D) http://orcid.org/0000-0003-4220-8638

Clara S Arias-Monsalve. Ph.D.

Corporación Universitaria Remington, Facultad de Medicina Veterinaria, Grupo GINVER, Medellín, Colombia

Clara.arias@uniremington.edu.co

(D) https://orcid.org/0000-0003-3875-1566

Daisy A. Gómez-R. M.Sc.

Corporación Universitaria Remington, Facultad de Medicina Veterinaria, Grupo GINVER, Medellín, Colombia.

*daisy.gomez@uniremington.edu.co

(D) https://orcid.org/0000-0002-5046-2989

\section{RESUMEN}

Las áreas periurbanas han sido documentadas como zonas de transición entre ambientes naturales y zonas urbanas las cuales son fundamentales para el mantenimiento de la avifauna asociada a las grandes urbes, albergando incluso altos niveles de riqueza y diversidad de especies. En el presente trabajo es documentado el ensamblaje de aves en una zona periurbana del municipio de Medellín (Colombia), la cual, en el último siglo ha sufrido una fuerte transformación de su cobertura natural y es considerada de alto interés para la conservación de los servicios ecosistémicos de la ciudad. El estudio se llevó a cabo en la sede de prácticas de la Corporación Universitaria Remington, ubicada en el corregimiento de Santa Elena. El registro de la avifauna se realizó mediante la captura de individuos con redes de niebla y la observación directa en recorridos libres, llevados a cabo en muestreos en agosto-septiembre de 2017, febrero-mayo de 2018 y septiembre de 2020. Adicionalmente, registros para algunas especies fueron obtenidos del muestreo con cámaras trampa, avistamientos ocasionales y datos sonoros. Se registraron un total de 54 especies de aves pertenecientes a 12 órdenes y 27 familias, incluidas dos especies endémicas, una casi endémica y dos especies migratorias. Este trabajo reporta la presencia de 35 especies que no habían sido previamente registradas en listados publicados para el corregimiento, principalmente

Como citar (Vancouver).

Agudelo-Rendón D, Rendón-Gutiérrez N, Cadavid-Ramírez AC, Choperena-Palencia MC, Arias-Monsalve CS, Gómez-R DA. Composición del ensamblaje de aves en una zona periurbana de Medellín, Colombia. Rev Colombiana Cienc Anim. Recia. 2020; 12(2):e782. https://doi.org/10.24188/recia.v12. n2.2020.782 
especies asociadas a áreas abiertas y zonas de bordes de bosque. Los resultados presentados resaltan la importancia de las áreas periurbanas en la diversidad de la avifauna local en las grandes ciudades.

Palabras claves: Aves urbanas; biodiversidad; conservación; Hypopyrrhus pyrohypogaster (Fuentes: MeSH, AGROVOC, CAB).

\begin{abstract}
Peri-urban areas have been documented as transitional zones between natural environments and urban areas, which are essential for the maintenance of the birdlife associated with large cities, even harboring high levels of species richness and diversity. In this work, the assemblages of birds in a peri-urban area of the municipality of Medellín (Colombia) is documented. This area has undergone a strong transformation of its natural cover in the last century and is considered of high interest for the conservation of ecosystem services on which the city depends. The study was carried out at the Remington University Veterinary Clinic, located in the town of Santa Elena. The avifauna records were obtained from capturing individuals with mist nets and direct observation in free walks carried out in August-September 2017, February-May 2018 and September 2020. Ad ditionally, records for some species were derived from camera trap sampling, occasional sightings, and sound data. A total of 54 bird species belonging to 12 orders and 27 families were recorded, including two endemic species, one near-endemic and two migratory species. This work reports the presence of 35 species that had not been previously registered in published lists for the township, mainly species associated with open areas and forest edges. Obtained results highlight the importance of peri-urban areas for the diversity of local birdlife in large cities.
\end{abstract}

Keywords: Biodiversity; Conservation; Hypopyrrhus pyrohypogaster; Urban birds, (Sources: MeSH, AGROVOC, CAB).

\title{
INTRODUCCIÓN
}

Los procesos de urbanización conllevan a una homogenización del paisaje y a la pérdida selectiva de cobertura boscosa, lo que impacta negativamente la diversidad en términos del número de especies, composición y estructura de las comunidades (1). A medida que avanza la frontera urbana, los espacios periurbanos se convierten en zonas de transición y de importancia para el mantenimiento de la biodiversidad presente en las urbes (2). Estos espacios llegan a ser sitios de conexión con las áreas naturales y en muchos casos mantienen valores altos de diversidad y riqueza, gracias a que albergan ensamblajes compuestos tanto por especies generalistas como especialistas en sus requerimientos de hábitat $(3,4)$.

La avifauna es documentada como uno de los grupos de vertebrados que responden rápidamente en términos de riqueza y abundancia a diferentes niveles de transformación de los hábitats naturales (3). Las características del paisaje han sido encontradas entre los aspectos de mayor influencia en la presencia de especies de aves en áreas urbanas del neotrópico $(5,6)$. En el caso de Colombia, la ocurrencia de especies en ambientes urbanos es limitada principalmente por la existencia de parques arbolados, jardines, lagos y una alta heterogeneidad de la estructura vegetal $(7,8,9,10,11)$.

El Valle de Aburrá, incluyendo el municipio de Medellín, conserva sobre sus laderas significativas extensiones de cobertura vegetal nativa conectadas con las áreas urbanas, las cuales han mostrado ser fundamentales en el mantenimiento de la avifauna regional $(10,12)$. Santa Elena, uno de los corregimientos de Medellín, es un área de marcada expansión urbana, vital en la provisión de servicios ecosistémicos para la ciudad y con áreas naturales albergando ensamblajes de aves de alta riqueza $(12,13)$. Estudios recientes, sin embargo, documentan una disminución marcada en el número de especies en los principales fragmentos de bosque de la zona, probablemente asociada a la fragmentación del hábitat y la pérdida de conectividad que actualmente enfrenta $(14,15)$. En este contexto, las áreas periurbanas podrían llegar a emerger como áreas importantes para el mantenimiento de la riqueza de la avifauna a escala local y regional (4). Como un aporte al conocimiento y conservación de la avifauna en ambientes urbanos, el presente trabajo presenta los resultados de la caracterización del ensamblaje de aves en una zona periurbana del Valle de Aburrá.

\section{MATERIALES Y MÉTODOS}

El estudio fue desarrollado en la sede de prácticas veterinarias de la Corporación Universidad Remington (CUR) (6.23907 $\mathrm{N}, 75.51438^{\circ} 0 ; 2.300 \mathrm{msnm}$ ), ubicada en el corregimiento de Santa Elena, zona periurbana del municipio de Medellín (departamento de Antioquia). La sede cuenta con un área de 70,400 $\mathrm{m}^{2}$ y está compuesta por terrenos destinados al pastoreo, donde predomina la especie herbácea Brachiaria sp., y algunos fragmentos de bosque secundario altamente 
intervenidos. El área que comprende la sede es atravesada por una vía de alto flujo vehicular que conecta la ciudad y el corregimiento de Santa Elena (Figura 1).
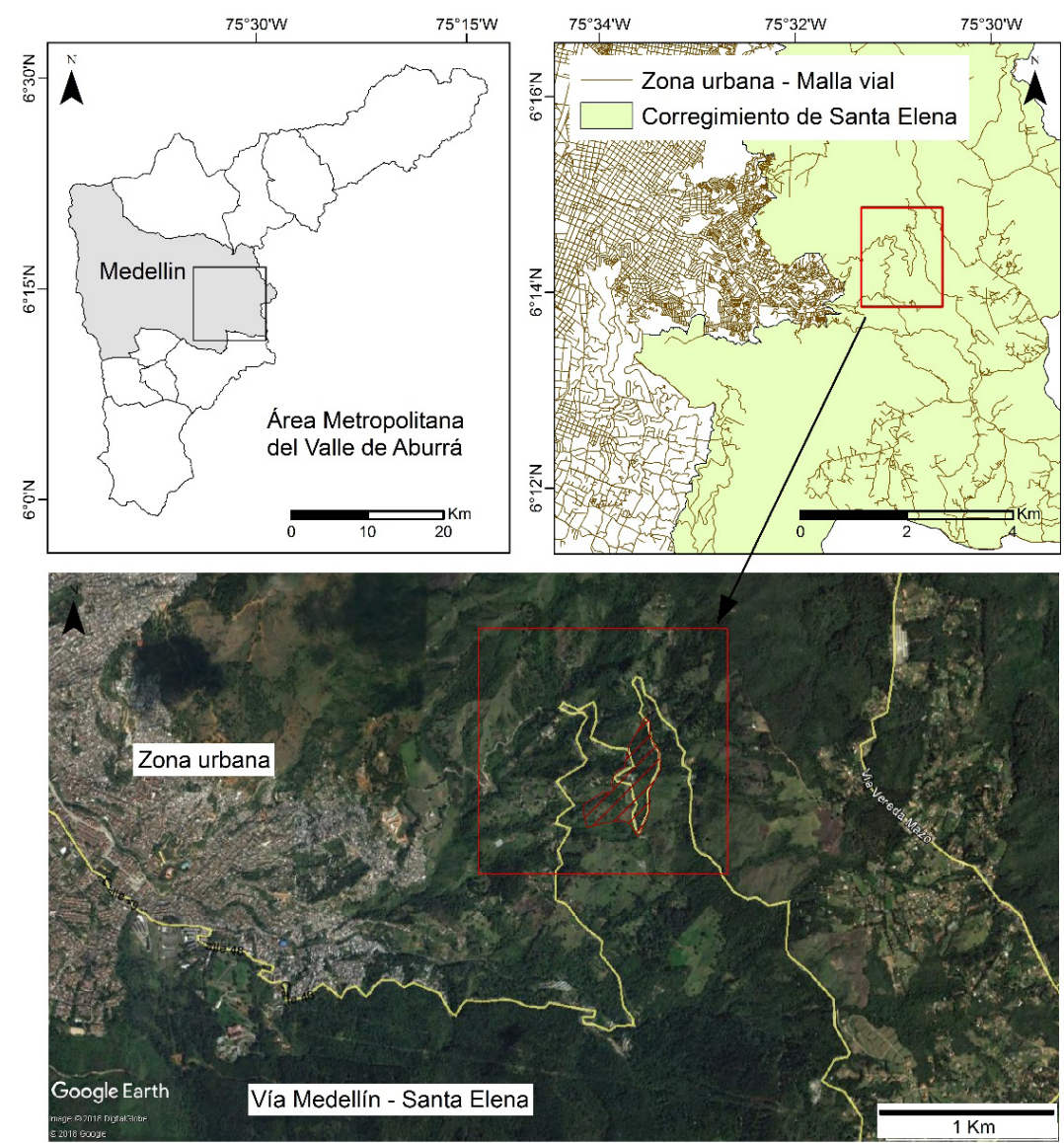

Figura 1. Localización geográfica del área de estudio en el corregimiento de Santa Elena, Medellín, Colombia. El área demarcada con líneas diagonales corresponde a la sede CUR (Fuentes: Google Earth, 2018; Base cartográfica de Medellín, Colombia).

Los registros de aves fueron obtenidos a través de la captura de individuos y la observación directa (16), en periodos de muestreo que comprendieron entre agosto y septiembre de 2017, febrero y mayo de 2018 y septiembre de 2020 . Para la captura de individuos se emplearon tres redes de niebla de $6 \mathrm{~m} \mathrm{x} 3$ m, invirtiendo un esfuerzo total de 69 horas-red. El avistamiento de individuos fue llevado a cabo mediante recorridos libres, acumulando un total de 52 horas de observación. Los muestreos fueron realizados durante tres a cinco días por mes, en jornadas alternas entre la mañana (6:00-10:00) y la tarde (15:00-18:00). Adicionalmente, para complementar el listado de especies, se incluyeron registros visuales y sonoros obtenidos fuera de los muestreos y algunos derivados de cámaras trampa instaladas en el área de estudio.

Para evaluar la representatividad del muestreo, se generó una curva de acumulación de especies a partir del número de muestras, empleando el estimador Mao Tao $\left(\mathrm{S}_{o b}\right)$. Adicionalmente, se emplearon los estimadores de riqueza Chao2, ICE, Jack 1 y Jack 2, basados en ocurrencia, para calcular el número esperado de especies. Las estimaciones solo incluyeron las especies registradas durante los muestreos estandarizados y cada muestra fue definida como 20 registros continuos, independiente del método con el que se hayan obtenido (16). Todos los análisis fueron realizados en el programa Estimates 9.1.0 (Colwell 2006).

\section{RESULTADOS}

Se registraron un total de 54 especies distribuidas en 12 órdenes y 27 familias, de la cuales 33 pertenecen al orden Paseriformes (Tabla 1). Las familias con la mayor riqueza fueron Thraupidae con 11 especies, Tyrannidae y Trochilidae con 5 y 4 respectivamente, y las demás familias estuvieron representadas por entre una y tres especies. Dentro de los registros se encontraron las especies endémicas Hypopyrrhus pyrohypogaster y Ortalis columbiana, y la especie casi endémica Stilpnia vitriolina. Adicionalmente, se registraron las especies migratorias boreales Piranga rubra y Catharus 
ustulatus. Cuarenta y seis de las especies fueron registradas principalmente mediante avistamiento directo, mientras los registros para seis fueron exclusivamente por cámaras trampa, una capturada y una por registro sonoro (Tabla 1).

Tabla 1. Lista de especies de aves presentes en la sede de prácticas de la Corporación Universitaria Remington en un área periurbana del Valle de Aburrá.

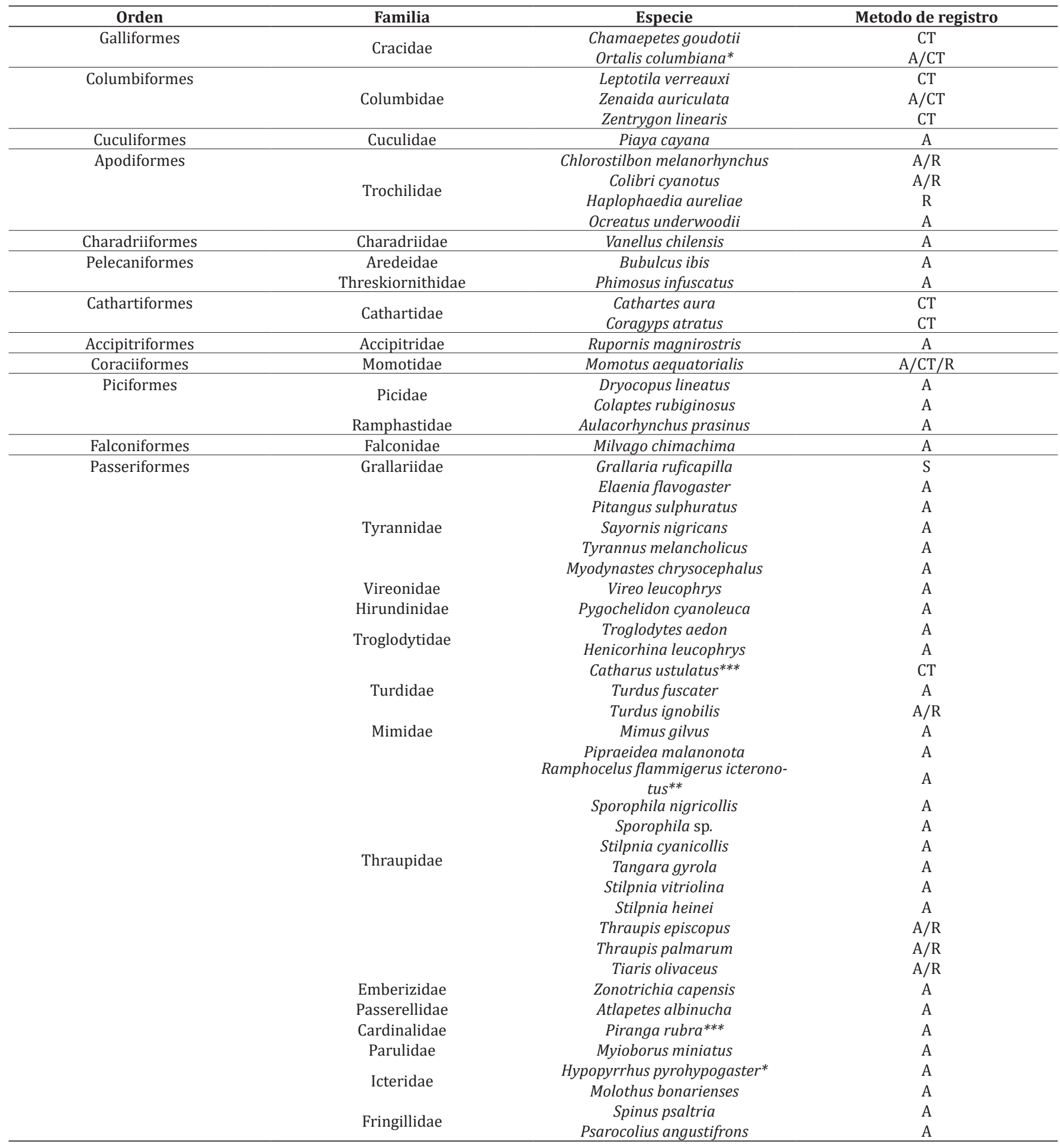

*Especie endémica. ${ }^{* *}$ Especie casi endémica. ${ }^{* * *}$ Especie migratoria.

Método de registro: A: Avistamiento; CT: Cámara trampa; R: Red de niebla; S: Registro sonoro.

Los registros obtenidos de los muestreos estandarizados correspondieron a 34 especies. La curva de acumulación basada en dichos registros evidencia que la tasa de acumulación de especies tiende a decrecer con el incremento en el número 
de muestras, pero sin alcanzar un comportamiento asintótico completo (Figura 2). Esto concuerda con los resultados de los estimadores basados en ocurrencia, los cuales indican una riqueza esperada para el área de estudio de entre 42 y 49 especies (Tabla 2). De esta menara, el muestreo alcanzó una representatividad estimada de entre el 68\% (Jack2) y el $80.9 \%$ (Chao2) de la riqueza.

Tabla 2. Riqueza de especies y estimadores de riqueza basados en ocurrencia ( \pm desviación estándar).

\begin{tabular}{ccc}
\hline Estimadores & & Riqueza \\
\hline Riqueza de Especies & $\mathrm{S}_{\text {obs }}$ & $34 \pm 2.9$ \\
Estimador de riqueza & Chao2 & $41.87 \pm 6.87$ \\
& ICE & $43.47 \pm 0$ \\
& Jack1 & $44.29 \pm 3.82$ \\
& Jack2 & $49.92 \pm 0$ \\
\hline
\end{tabular}

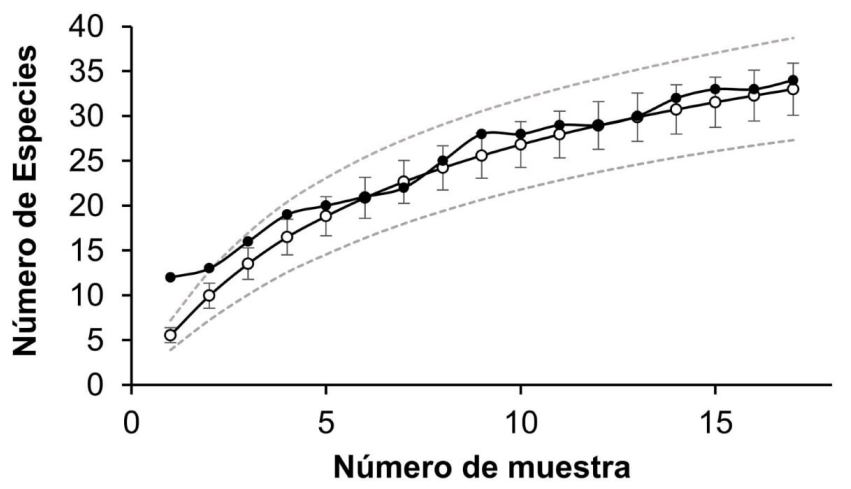

Figura 2. Curva de acumulación de especies. Puntos negros: riqueza observada; puntos blancos: riqueza esperada (estimados $\mathrm{S}_{\text {obs }}$ ); líneas punteadas: intervalos de confianza del 95\%; barras: desviación estándar.

\section{DISCUSIÓN}

Los resultados presentados en este trabajo resaltan la importancia que las zonas periurbanas podrían llegar a tener en el mantenimiento de la diversidad de aves en las grandes ciudades. A pesar de su extensión (7 ha), se encontró que la riqueza de especies registrada en la sede CUR es mayor a la reportada previamente en fragmentos de bosque aislados en otras zonas del corregimiento (28-49 especies), los cuales alcanzan extensiones mínimas de 41 ha (13). Igualmente, cuando se compara la composición de especies con otras localidades cercanas, se encuentra que del presente estudio 35 especies no habían sido previamente reportadas en los listados publicados para el corregimiento (13,15). Algunas de estas especies corresponden a Leptotila verreauxi, Chlorostilbon melanorhynchus, Elaenia flavogaster, Myodynastes chrysocephalus, Troglodytes aedon, Turdus ignobilis, Pipraeidea malanonota, Stilpnia heinei.

El ensamblaje de aves en la sede CUR fue dominada por especies de áreas abiertas y bordes de bosques. Este hallazgo refleja la estructura del hábitat presente en la zona, donde se encuentran pastos limpios y pastos arbolados delimitados por unos pocos remanentes de bosque en los bordes de las quebradas. Dentro de los registros obtenidos se encuentran especies comunes de áreas urbanas y periurbanas como Thraupis episcopus, Zenaida auriculata, Pitangus sulphuratus y Zonotrichia capensis $(17,18)$. Estas especies tienen una alta capacidad para desarrollarse en diferentes tipos de ambientes y pueden hacer uso de diferentes recursos de acuerdo con su disponibilidad (19). De las once especies registradas para la familia Thraupidae, nueve son consideradas generalistas y aprovechan mejor los hábitats abiertos y bordes de bosque (20). Otras especies de esta familia reportadas para zonas cercanas no fueron registradas, posiblemente porque los fragmentos de bosque presentes corresponden a franjas riparias de menos de $15 \mathrm{~m}$ de ancho y con variabilidad en la disponibilidad de frutos ornitócoros. A pesar de no haberse realizado un estudio sistemático sobre la disponibilidad de alimento para las aves, es de esperarse una disminución en los recursos de estos bosques debido a su reducida extensión, la tala selectiva que han sufrido y la baja conectividad que exhiben (21). 
Los estudios de diversidad de aves en zonas periurbanas son básicos para comprender los efectos de la transformación del paisaje sobre los ambientes naturales y sus comunidades, y pueden facilitar y guiar la toma de medidas de conservación en zonas sujetas a presiones derivadas de la transformación urbanística (22). La preservación, restauración y enriquecimiento vegetal de las zonas periurbanas puede permitir la recolonización de especies nativas y contribuir a el mantenimiento de la biodiversidad y los servicios ambientales que estas áreas prestan a las grandes urbes (23).

\section{Conflicto de intereses}

Los autores declaran que no existen conflictos de intereses con el presente trabajo.

\section{Agradecimientos}

Agradecemos a la Facultad de Medicina Veterinaria y al personal de la Clínica Veterinaria Uniremington en Santa Elena por permitir y apoyar el desarrollo de este proyecto.

\section{Financiación}

Esta investigación fue financiada por la Vicerrectoría de Investigaciones de la corporación Universitaria Remington e hizo parte del proyecto “Caracterización de la Fauna Silvestre en la sede CUR Santa Elena” con código 4000000048-16.

\section{REFERENCIAS}

1. McKinney M. Effects of urbanization on species richness: A review of plants and animals. Urban Ecosyst. 2008; 11(2):161-176. https://doi.org/10.1007/s11252-007-0045-4

2. Blair R, Johnson E. Suburban habitats and their role for birds in the urban-rural habitat network: points of local invasion and extinction?. Landsc Ecol. 2008; 23:1157-1169. https://doi.org/10.1007/s10980-008-9267-y

3. Blair R. Birds and Butterflies along an Urban Gradient: Surrogate Taxa for Assessing Biodiversity?. Ecol Appl. 1999; 9(1):164-170. https://doi.org/10.2307/2641176

4. Blair R. Land use and avian species diversity along an urban gradient. Ecol Appl. 1996; 6(2):506-519. https://doi. org/10.2307/2269387

5. Silva C, Garcia C, Estay S, Barbosa O. Bird Richness and abundance in response to urban form in a latin American City: Valdivia, Chile as a case study. PLoS One. 2015; 10(9):e0138120. https://doi.org/10.1371/journal.pone.0138120

6. Caula S, Giner S, De Nóbrega J. Aves urbanas: un estudio comparativo en dos parques tropicales con diferente grado de intervención humana (Valencia, Venezuela). FARAUTE Cs. Tecnol. 2010; 5(2):1-13. http://servicio.bc.uc.edu.ve/ facyt/vol5n2/art02.pdf

7. Muñoz M, Fierro-Calderón K, Rivera-Gutierrez H. Las aves del campus de la Universidad del Valle, una isla verde urbana en Cali, Colombia. Ornitol Colomb. 2007; 5(5):5-20. https://asociacioncolombianadeornitologia.org/wpcontent/uploads/revista/oc5/universidaddelvalle5-20.pdf

8. Rivera G. Composición y estructura de una comunidad de aves en un área suburbana en el suroccidente colombiano. Ornitol Colomb. 2006; 4:28-38. https://asociacioncolombianadeornitologia.org/wp-content/uploads/revista/oc4/ Suburbana.pdf

9. Marín-Gómez O. Avifauna del campus de la Universidad del Quindío. Boletín SA0. 2005; 15(2):42-60. http://www. sao.org.co/publicaciones/boletinsao/06-Marin-AvifaunaQuindio.pdf

10. Vásquez J, Castaño G. Identificación de áreas prioritarias para la conservación de la avifauna en la zona urbana del municipio de Medellín, Colombia. Bol Cient Mus Hist Nat s. 2008; 12:51-61. http://www.scielo.org.co/pdf/bccm/ v12n1/v12n1a04.pdf 
11. Rivera V. Inventario de aves en el campus del Tecnológico de Antioquia sede Robledo Medellín. Cuaderno Activa. 2016; (9):53-63. https://ojs.tdea.edu.co/index.php/cuadernoactiva/article/view/420/492

12. Garizabal-Carmona J, Gutiérrez-Vásquez C, David S. Diversidad de aves en cuatro localidades con bosques fragmentados en el municipio de Medellín. En: Gutierrez-Vasquez C, Osorio-Velez L, editores. Mas Bosques para Medellín-Sembrando árboles para la vida. $1^{\text {a }}$ ed. Colombia: Fundacion CIPAV y Alcaldia de Medellin; 2014. https://www.researchgate.net/publication/313091095 Diversidad de aves en cuatro localidades con bosques fragmentados en el municipio de Medellin

13. Castaño-Villa G, Patiño-Zabala J. Composición de la comunidad de aves en bosques fragmentados en la región de Santa Elena, Andes centrales colombianos. Bol Cient Mus Hist Nat. 2007; 11:47-60. http://www.scielo.org.co/pdf/ bccm/v11n1/v11n1a03.pdf

14. Castaño-Villa G, Patiño-Zabala J. Cambios en la composición de la avifauna en Santa Helena durante el siglo XX. Cron For Medio Ambient. 2000; 15:137-161. https://www.researchgate.net/publication/237022908_Cambios en la composicion de la avifauna en Santa Helena durante el siglo XX

15. Castaño-Villa G, Patiño-Zabala J. Extinciones locales de aves en fragmentos de bosque de la región de Santa Elena, Andes centrales, Colombia. Hornero. 2008; 23(1):23-34. https://bibliotecadigital.exactas.uba.ar/download/ hornero/hornero v023 n01 p023.pdf

16. Villareal H, Álvarez M, Córdoba-Córdoba S, Escobar F, Fagua G, Gast F, et al. Manual de métodos para el desarrollo de inventarios de biodiversidad. $1^{a}$ Edición. Colombia: Instituto de Investigación de Recursos Biológicos Alexander von Humboldt; 2004.

17. Martins-Oliveira L, Leal-Marques R, Nunes C, Franchin A, Júnior O. Forrageamento de Pitangus sulphuratus e de Tyrannus melancholicus (Aves: Tyrannidae) em hábitats urbanos. Biosci J. 2012; 28(6):1038-1050. http://www. seer.ufu.br/index.php/biosciencejournal/article/view/14404/11130

18. Osorio-Méndez J, Marín-Gómez O. Comportamiento de forrajeo del Azulejo Común (Thraupis episcopus) y el Azulejo Palmero (T. palmarum) en un área urbana en la Cordillera Central de Colombia. Boletin SAO. 2016; 25(1-2):12-16. https://sao.org.co/publicaciones/boletinsao/25 1n2/evNC3 BS25 2016.pdf

19. Londoño-Betancourth J. Una mirada a la diversidad ornitológica de Pereira. Bol Cient Mus Hist Nat. 2011; 15(1):84103. http://www.scielo.org.co/pdf/bccm/v15n1/v15n1a07.pdf

20. Rivera G. Composición y estructura de una comunidad de aves en un área suburbana en el suroccidente colombiano. Ornitol Colomb. 2006; 4:28-38. https://asociacioncolombianadeornitologia.org/wp-content/uploads/revista/oc4/ Suburbana.pdf

21. Colorado-Zuluaga G, Vásquez J, Mazo I. Modelo de conectividad ecológica de fragmentos de bosque andino en Santa Elena (Medellín, Colombia). Acta Biológica Colombiana. 2017; 22(3):379-393. https://doi.org/10.15446/abc. $\underline{\mathrm{v} 22 \mathrm{n} 3.63013}$

22. Padoa-Schioppa E, Baietto M, Massa R, Bottoni L. Bird communities as bioindicators: The focal species concept in agricultural landscapes. Ecol Indic. 2006; 6(1):83-93. https://doi.org/10.1016/j.ecolind.2005.08.006

23. Montealegre-Talero C, Echeverry-Galvis M, Renjifo L. Restored corridors as potencial habitat for resident bird species in the Central Andes of Colombia. Ornitol Colomb. 2017; 16:eA07. https://asociacioncolombianadeornitologia.org/ wp-content/uploads/2018/10/16eA0701-13.pdf 